\title{
AN ESTIMATE ON RIEMANNIAN MANIFOLDS OF DIMENSION 4.
}

\author{
SAMY SKANDER BAHOURA
}

\begin{abstract}
We give an estimate of type $\sup \times$ inf on Riemannian manifold of dimension 4 for a Yamabe type equation.
\end{abstract}

\section{Mathematics Subject Classification: 53C21, 35J60 35B45 35B50}

\section{Introduction AND MAin RESUlts}

In this paper, we deal with the following Yamabe type equation in dimension $n=4$ :

$$
\Delta_{g} u+h u=8 u^{3}, u>0,
$$

Here, $\Delta_{g}$ is the Laplace-Beltrami operator and $h$ is an arbitrary bounded function..

The equation (11) was studied a lot, when $M=\Omega \subset \mathbb{R}^{n}$ or $M=\mathbb{S}_{n}$ see for example, [2-4], [11], [15]. In this case we have a sup $\times$ inf inequality. The corresponding equation in two dimensions on open set $\Omega$ of $\mathbb{R}^{2}$, is:

$$
\Delta u=V(x) e^{u}
$$

The equation (2) was studied by many authors and we can find very important result about a priori estimates in [8], [9], [12], [16], and [19]. In particular in [9] we have the following interior estimate:

$$
\sup _{K} u \leq c=c\left(\inf _{\Omega} V,\|V\|_{L^{\infty}(\Omega)}, \inf _{\Omega} u, K, \Omega\right) .
$$

And, precisely, in [8], [12], [16], and [20], we have:

$$
C \sup _{K} u+\inf _{\Omega} u \leq c=c\left(\inf _{\Omega} V,\|V\|_{L^{\infty}(\Omega)}, K, \Omega\right),
$$

and,

$$
\sup _{K} u+\inf _{\Omega} u \leq c=c\left(\inf _{\Omega} V,\|V\|_{C^{\alpha}(\Omega)}, K, \Omega\right) .
$$

where $K$ is a compact subset of $\Omega, C$ is a positive constant which depends on $\frac{\inf _{\Omega} V}{\sup _{\Omega} V}$, and, $\alpha \in(0,1]$. When $6 h=R_{g}$ the scalar curvature, and $M$ compact, the equation (11) is Yamabe equation. T. Aubin and R. Schoen have proved the existence of solution in this case, see for example [1] and [14] for a complete and detailed summary. When $M$ is a compact Riemannian manifold, there exist some compactness result for equation (1) see [18]. Li and Zhu see [18],

Date: March 2, 2023. 
proved that the energy is bounded and if we suppose $M$ not diffeormorfic to the three sphere, the solutions are uniformly bounded. To have this result they use the positive mass theorem. Now, if we suppose $M$ Riemannian manifold (not necessarily compact) Li and Zhang [17] proved that the product sup $\times$ inf is bounded. Here we extend the result of [5]. Our proof is an extension Li-Zhang result in dimension 3, see [3] and [17], and, the moving-plane method is used to have this estimate. We refer to Gidas-Ni-Nirenberg for the moving-plane method, see [13]. Also, we can see in [3, $6,11,16,17,10]$, some applications of this method, for example an uniqueness result. We refer to [7] for the uniqueness result on the sphere and in dimension 3. Here, we give an equality of type sup $\times$ inf for the equation (11) in dimension 4 . In dimension greater than 3 we have other type of estimates by using moving-plane method, see for example $[3,5]$. There are other estimates of type sup + inf on complex Monge-Ampere equation on compact manifolds, see [20-21] . They consider, on compact Kahler manifold $(M, g)$, the following equation:

$$
\left\{\begin{array}{l}
\left(\omega_{g}+\partial \bar{\partial} \varphi\right)^{n}=e^{f-t \varphi} \omega_{g}^{n} \\
\omega_{g}+\partial \bar{\partial} \varphi>0 \text { on } M
\end{array}\right.
$$

And, they prove some estimates of type $\sup _{M}+m \inf _{M} \leq C$ or $\sup _{M}+m \inf _{M} \geq C$ under the positivity of the first Chern class of M. Here, we have,

Theorem 1.1. For all compact set $K$ of $M$, there is a positive constant $c$, which depends only on, $h_{0}=\|h\|_{L^{\infty}(M)}, K, M, g$ such that:

$$
\left(\sup _{K} u\right)^{1 / 3} \times \inf _{M} u \leq c,
$$

for all u solution of (1).

This theorem extend to the dimension 4 a result of the author and of $\mathrm{Li}$ and Zhang result, see [17] . Here, we use a different method than the method of Li and Zhang in [17]. Also, we extend a result of [5].

Corollary 1.2. For all compact set $K$ of $M$ there is a positive constant $c$, such that:

$$
\sup _{K} u \leq c=c\left(g, m, h_{0}, K, M\right) \text { if } \inf _{M} u \geq m>0,
$$

for all u solution of (1).

\section{PRoOF OF THE RESUlts}

\section{Proof of theorem 1.1:}

Let $x_{0}$ be a point of $M$. We want to prove a uniform estimate around $x_{0}$.

Let $\left(u_{i}\right)_{i}$ be a sequence of solutions to: 


$$
\Delta u_{i}+h u_{i}=8 u_{i}^{3}, u_{i}>0
$$

We argue by contradiction, we assume that the $\sup \times$ inf is not bounded.

$\forall c, R>0 \exists u_{c, R}$ solution to (1) such that:

$$
R^{2}\left(\sup _{B\left(x_{0}, R\right)} u_{c, R}\right)^{1 / 3} \times \inf _{B\left(x_{0}, 2 R\right)} u_{c, R} \geq c
$$

Proposition 2.1. (blow-up analysis)

There is a sequence of points $\left(y_{i}\right)_{i}, y_{i} \rightarrow x_{0}$ and two sequences of positive real numbers $\left(l_{i}\right)_{i},\left(L_{i}\right)_{i}, l_{i} \rightarrow 0, L_{i} \rightarrow+\infty$, such that if we set $v_{i}(y)=\frac{u_{i}\left[\exp _{y_{i}}\left(y /\left[u_{i}\left(y_{i}\right)\right]\right)\right]}{u_{i}\left(y_{i}\right)}$, we have:

$$
\begin{gathered}
0<v_{i}(y) \leq \beta_{i} \leq 2, \beta_{i} \rightarrow 1 . \\
v_{i}(y) \rightarrow \frac{1}{1+|y|^{2}}, \text { uniformly on compact sets of } \mathbb{R}^{4} . \\
l_{i}^{2}\left(u_{i}\left(y_{i}\right)\right)^{1 / 3} \min _{M} u_{i} \rightarrow+\infty .
\end{gathered}
$$

\section{Proof:}

We use the hypothesis (44), we take two sequences, $R_{i}>0, R_{i} \rightarrow 0$ and $c_{i} \rightarrow+\infty$, such that,

$$
R_{i}^{2}\left(\sup _{B\left(x_{0}, R_{i}\right)} u_{i}\right)^{1 / 3} \times \inf _{B\left(x_{0}, 2 R_{i}\right)} u_{i} \geq c_{i} \rightarrow+\infty
$$

Let, $x_{i} \in B\left(x_{0}, R_{i}\right)$, such that $\sup _{B\left(x_{0}, R_{i}\right)} u_{i}=u_{i}\left(x_{i}\right)$ and $s_{i}(x)=\left[R_{i}-d\left(x, x_{i}\right)\right] u_{i}(x), x \in$ $B\left(x_{i}, R_{i}\right)$. Then, $x_{i} \rightarrow x_{0}$.

We have:

$$
\max _{B\left(x_{i}, R_{i}\right)} s_{i}(x)=s_{i}\left(y_{i}\right) \geq s_{i}\left(x_{i}\right)=R_{i} u_{i}\left(x_{i}\right) \geq \sqrt{c_{i}} \rightarrow+\infty
$$

We set : 


$$
l_{i}=R_{i}-d\left(y_{i}, x_{i}\right), \bar{u}_{i}(y)=u_{i}\left[\exp _{y_{i}}(y)\right], v_{i}(z)=\frac{u_{i}\left[\exp _{y_{i}}\left(z /\left[u_{i}\left(y_{i}\right)\right]\right)\right]}{u_{i}\left(y_{i}\right)}
$$

Clearly, we have, $y_{i} \rightarrow x_{0}$. We obtain:

$$
L_{i}=\frac{l_{i}}{\left(c_{i}\right)^{1 / 4}}\left[u_{i}\left(y_{i}\right)\right]=\frac{\left[s_{i}\left(y_{i}\right)\right]}{c_{i}^{1 / 4}} \geq \frac{c_{i}^{1 / 2}}{c_{i}^{1 / 4}}=c_{i}^{1 / 4} \rightarrow+\infty
$$

If $|z| \leq L_{i}$, then $y=\exp _{y_{i}}\left[z /\left[u_{i}\left(y_{i}\right)\right]\right] \in B\left(y_{i}, \delta_{i} l_{i}\right)$ with $\delta_{i}=\frac{1}{\left(c_{i}\right)^{1 / 4}}$ and $d\left(y, y_{i}\right)<R_{i}-$ $d\left(y_{i}, x_{i}\right)$, thus, $d\left(y, x_{i}\right)<R_{i}$ and, $s_{i}(y) \leq s_{i}\left(y_{i}\right)$. We can write,

$$
u_{i}(y)\left[R_{i}-d\left(y, y_{i}\right)\right] \leq u_{i}\left(y_{i}\right) l_{i}
$$

But, $d\left(y, y_{i}\right) \leq \delta_{i} l_{i}, R_{i}>l_{i}$ and $R_{i}-d\left(y, y_{i}\right) \geq R_{i}-\delta_{i} l_{i}>l_{i}-\delta_{i} l_{i}=l_{i}\left(1-\delta_{i}\right)$, hence, we obtain,

$$
0<v_{i}(z)=\frac{u_{i}(y)}{u_{i}\left(y_{i}\right)} \leq \frac{l_{i}}{l_{i}\left(1-\delta_{i}\right)} \leq 2
$$

We set, $\beta_{i}=\frac{1}{1-\delta_{i}}$, clearly $\beta_{i} \rightarrow 1$.

The function $v_{i}$ satisfies the following equation:

$$
-g^{j k}(z) \partial_{j k} v_{i}-\partial_{k}\left[g^{j k} \sqrt{|g|}\right](z) \partial_{j} v_{i}+\frac{h(z)}{\left[u_{i}\left(y_{i}\right)\right]^{2}} v_{i}=8 v_{i}^{3}
$$

We use Ascoli and Ladyzenskaya theorems to obtain the local uniform convergence (on every compact set of $\mathbb{R}^{4}$ ) of $\left(v_{i}\right)_{i}$ to $v$ solution on $\mathbb{R}^{4}$ to:

$$
\Delta v=8 v^{3}, v(0)=1,0 \leq v \leq 1 \leq 2
$$

By the maximum principle, we have $v>0$ on $\mathbb{R}^{n}$. According to Caffarelli-Gidas-Spruck result (see [10]), we have, $v(y)=\frac{1}{1+|y|^{2}}$.

\section{$\underline{\text { Polar Geodesic Coordinates }}$}


Let $u$ be a function on $M$. We set $\bar{u}(r, \theta)=u\left[\exp _{x}(r \theta)\right]$. We denote $g_{x, i j}$ the local expression of the metric $g$ in the exponential chart centered at $x$.

We set,

$$
\begin{gathered}
w_{i}(t, \theta)=e^{t} \bar{u}_{i}\left(e^{t}, \theta\right)=e^{t} u_{i}\left[\exp _{y_{i}}\left(e^{t} \theta\right)\right], \\
a\left(y_{i}, t, \theta\right)=\log J\left(y_{i}, e^{t}, \theta\right)=\log \left[\sqrt{\operatorname{det}\left(g_{y_{i}, i j}\right)}\right]
\end{gathered}
$$

We can write the Laplace-Beltrami operator in polar geodesic coordinates:

$$
-\Delta u=\partial_{r r} \bar{u}+\frac{3}{r} \partial_{r} \bar{u}+\partial_{r}[\log J(x, r, \theta)] \partial_{r} \bar{u}-\frac{1}{r^{2}} \Delta_{\theta} \bar{u}
$$

We deduce the two following lemmas:

Lemma 2.2. The function $w_{i}$ is a solution to:

$$
-\partial_{t t} w_{i}-\partial_{t} a \partial_{t} w_{i}-\Delta_{\theta} w_{i}+c w_{i}=8 w_{i}^{3}
$$

with

$$
c=c\left(y_{i}, t, \theta\right)=1+\partial_{t} a+h e^{2 t}
$$

\section{Proof:}

We write:

$$
\begin{gathered}
\partial_{t} w_{i}=e^{2 t} \partial_{r} \bar{u}_{i}+w_{i}, \partial_{t t} w_{i}=e^{3 t}\left[\partial_{r r} \bar{u}_{i}+\frac{3}{e^{t}} \partial_{r} \bar{u}_{i}\right]+w_{i} . \\
\partial_{t} a=e^{t} \partial_{r} \log J\left(y_{i}, e^{t}, \theta\right), \partial_{t} a \partial_{t} w_{i}=e^{3 t}\left[\partial_{r} \log J \partial_{r} \bar{u}_{i}\right]+\partial_{t} a w_{i} .
\end{gathered}
$$

Lemma 1 follows.

Let $b_{1}\left(y_{i}, t, \theta\right)=J\left(y_{i}, e^{t}, \theta\right)>0$. We can write: 


$$
-\frac{1}{\sqrt{b_{1}}} \partial_{t t}\left(\sqrt{b_{1}} w_{i}\right)-\Delta_{\theta} w_{i}+\left[c(t)+b_{1}^{-1 / 2} b_{2}(t, \theta)\right] w_{i}=8 w_{i}{ }^{3},
$$

where, $b_{2}(t, \theta)=\partial_{t t}\left(\sqrt{b_{1}}\right)=\frac{1}{2 \sqrt{b_{1}}} \partial_{t t} b_{1}-\frac{1}{4\left(b_{1}\right)^{3 / 2}}\left(\partial_{t} b_{1}\right)^{2}$.

We set,

$$
\tilde{w}_{i}=\sqrt{b_{1}} w_{i}
$$

Lemma 2.3. The function $\tilde{w}_{i}$ is a solution to:

$$
\begin{gathered}
-\partial_{t t} \tilde{w}_{i}+\Delta_{\theta}\left(\tilde{w}_{i}\right)+2 \nabla_{\theta}\left(\tilde{w}_{i}\right) \cdot \nabla_{\theta} \log \left(\sqrt{b_{1}}\right)+\left(c+b_{1}^{-1 / 2} b_{2}-c_{2}\right) \tilde{w}_{i}= \\
=8\left(\frac{1}{b_{1}}\right) \tilde{w}_{i}^{3},
\end{gathered}
$$

where, $c_{2}$ is a function to be determined.

\section{Proof:}

We have:

$$
-\partial_{t t} \tilde{w}_{i}-\sqrt{b_{1}} \Delta_{\theta} w_{i}+\left(c+b_{2}\right) \tilde{w}_{i}=8\left(\frac{1}{b_{1}}\right) \tilde{w}_{i}^{3}
$$

But,

$$
\Delta_{\theta}\left(\sqrt{b_{1}} w_{i}\right)=\sqrt{b_{1}} \Delta_{\theta} w_{i}-2 \nabla_{\theta} w_{i} \cdot \nabla_{\theta} \sqrt{b_{1}}+w_{i} \Delta_{\theta}\left(\sqrt{b_{1}}\right),
$$

and,

$$
\nabla_{\theta}\left(\sqrt{b_{1}} w_{i}\right)=w_{i} \nabla_{\theta} \sqrt{b_{1}}+\sqrt{b_{1}} \nabla_{\theta} w_{i}
$$

we can write,

$$
\nabla_{\theta} w_{i} . \nabla_{\theta} \sqrt{b_{1}}=\nabla_{\theta}\left(\tilde{w}_{i}\right) \cdot \underset{6}{\nabla_{\theta}} \log \left(\sqrt{b_{1}}\right)-\tilde{w}_{i}\left|\nabla_{\theta} \log \left(\sqrt{b_{1}}\right)\right|^{2}
$$


we deduce,

$$
\sqrt{b_{1}} \Delta_{\theta} w_{i}=\Delta_{\theta}\left(\tilde{w}_{i}\right)+2 \nabla_{\theta}\left(\tilde{w}_{i}\right) \cdot \nabla_{\theta} \log \left(\sqrt{b_{1}}\right)-c_{2} \tilde{w}_{i}
$$

with $c_{2}=\left[\frac{1}{\sqrt{b_{1}}} \Delta_{\theta}\left(\sqrt{b_{1}}\right)+\left|\nabla_{\theta} \log \left(\sqrt{b_{1}}\right)\right|^{2}\right]$. Lemma 2 is proved.

\section{The moving-Plane method:}

Let $\xi_{i}$ be a real number, we assume $\xi_{i} \leq t$. We set $t^{\xi_{i}}=2 \xi_{i}-t$ and $\tilde{w}_{i}^{\xi_{i}}(t, \theta)=\tilde{w}_{i}\left(t^{\xi_{i}}, \theta\right)$. Set, $\lambda_{i}=-\log u_{i}\left(y_{i}\right)$

Proposition 2.4. We claim:

$$
\tilde{w}_{i}\left(\lambda_{i}, \theta\right)-\tilde{w}_{i}\left(\lambda_{i}+4, \theta\right) \geq \tilde{k}>0, \forall \theta \in \mathbb{S}_{3}
$$

For all $\beta>0$, there exists $c_{\beta}>0$ such that:

$$
\frac{1}{c_{\beta}} e^{t} \leq \tilde{w}_{i}\left(\lambda_{i}+t, \theta\right) \leq c_{\beta} e^{t}, \forall t \leq \beta, \forall \theta \in \mathbb{S}_{3}
$$

\section{Proof:}

As in [2], we have, $w_{i}\left(\lambda_{i}, \theta\right)-w_{i}\left(\lambda_{i}+4, \theta\right) \geq k>0$ for $i$ large, $\forall \theta$. We can remark that $b_{1}\left(y_{i}, \lambda_{i}, \theta\right) \rightarrow 1$ and $b_{1}\left(y_{i}, \lambda_{i}+4, \theta\right) \rightarrow 1$ uniformly in $\theta$, we obtain the first claim of proposition 2.4. For the second claim we use proposition 2.1, see also [2].

We set:

$$
\bar{Z}_{i}=-\partial_{t t}(\ldots)+\Delta_{\theta}(\ldots)+2 \nabla_{\theta}(\ldots) \cdot \nabla_{\theta} \log \left(\sqrt{b_{1}}\right)+\left(c+b_{1}^{-1 / 2} b_{2}-c_{2}\right)(\ldots)
$$

Remark : In the operator $\bar{Z}_{i}$, we can remark that:

$$
c+b_{1}^{-1 / 2} b_{2}-c_{2} \geq k^{\prime}>0, \text { for } t<<0,
$$

we can apply the maximum principle and the Hopf lemma.

\section{Goal:}


Like in [2], we have an elliptic second order operator. Here it is $\bar{Z}_{i}$, the goal is to use the "moving-plane" method to have a contradiction. For this, we must have:

$$
\bar{Z}_{i}\left(\tilde{w}_{i}^{\xi_{i}}-\tilde{w}_{i}\right) \leq 0 \text {, if } \tilde{w}_{i}^{\xi_{i}}-\tilde{w}_{i} \leq 0
$$

We write, $\Delta_{\theta}=\Delta_{g_{y_{i}, e^{t}, \mathbb{S}_{n-1}}}$. We obtain:

$$
\begin{gathered}
\bar{Z}_{i}\left(\tilde{w}_{i}^{\xi_{i}}-\tilde{w}_{i}\right)=\left(\Delta_{y_{i}, e^{\xi^{\xi_{i}}, \mathbb{S}_{3}}}-\Delta_{g_{y_{i}, e^{t}, \mathbb{S}_{3}}}\right)\left(\tilde{w}_{i}^{\xi_{i}}\right)+ \\
+2\left(\nabla_{\theta, e^{\xi_{i}}}-\nabla_{\theta, e^{t}}\right)\left(w_{i}^{\xi_{i}}\right) \cdot \nabla_{\theta, e^{\xi_{i}}} \log \left(\sqrt{b_{1}^{\xi_{i}}}\right)+2 \nabla_{\theta, e^{t}}\left(\tilde{w}_{i}^{\xi_{i}}\right) \cdot \nabla_{\theta, e^{t} \xi_{i}}\left[\log \left(\sqrt{b_{1}^{\xi_{i}}}\right)-\log \sqrt{b_{1}}\right]+ \\
+2 \nabla_{\theta, e^{t}} w_{i}^{\xi_{i}} \cdot\left(\nabla_{\theta, e^{\xi^{\xi_{i}}}}-\nabla_{\theta, e^{t}}\right) \log \sqrt{b_{1}}-\left[\left(c+b_{1}^{-1 / 2} b_{2}-c_{2}\right)^{\xi_{i}}-\left(c+b_{1}^{-1 / 2} b_{2}-c_{2}\right)\right] \tilde{w}_{i}^{\xi_{i}}+ \\
+8\left(\frac{1}{b_{1}^{\xi_{i}}}\right)\left(\tilde{w}_{i}^{\xi_{i}}\right)^{3}-8\left(\frac{1}{b_{1}}\right) \tilde{w}_{i}^{3} .
\end{gathered}
$$

Clearly, we have the following lemma:

\section{Lemma 2.5.}

$$
\begin{gathered}
b_{1}\left(y_{i}, t, \theta\right)=1-\frac{1}{3} \operatorname{Ricci}_{y_{i}}(\theta, \theta) e^{2 t}+\ldots, \\
R_{g}\left(e^{t} \theta\right)=R_{g}\left(y_{i}\right)+<\nabla R_{g}\left(y_{i}\right) \mid \theta>e^{t}+\ldots
\end{gathered}
$$

According to proposition 1 and lemma 3,

\section{Proposition 2.6.}

$$
\begin{gathered}
\bar{Z}_{i}\left(\tilde{w}_{i}^{\xi_{i}}-\tilde{w}_{i}\right) \leq 8\left(b_{1}^{\xi_{i}}\right)\left[\left(\tilde{w}_{i}^{\xi_{i}}\right)^{3}-\tilde{w}_{i}^{3}\right]++C\left|e^{2 t}-e^{2 t \xi_{i}}\right|\left(\left|\nabla_{\theta} \tilde{w}_{i}^{\xi_{i}}\right|+\left|\nabla_{\theta}^{2}\left(\tilde{w}_{i}^{\xi_{i}}\right)\right|\right)+ \\
+C\left|e^{2 t}-e^{2 t \xi_{i}}\right|\left(\left|R i c c i_{y_{i}}\right|+|h|\right) \tilde{w}_{i}^{\xi_{i}}+C^{\prime} w_{i}^{\xi_{i}}\left|e^{3 t^{\xi_{i}}}-e^{3 t}\right| .
\end{gathered}
$$

\section{Proof of proposition 2.6:}

In polar geodesic coordinates (and the Gauss lemma): 


$$
g=d t^{2}+r^{2} \tilde{g}_{i j}^{k} d \theta^{i} d \theta^{j} \text { et } \sqrt{\left|\tilde{g}^{k}\right|}=\alpha^{k}(\theta) \sqrt{\left[\operatorname{det}\left(g_{x, i j}\right)\right]}
$$

where $\alpha^{k}$ is the volume element of the unit sphere associated to $U^{k}$.

We can write (with lemma 2.3):

$$
\left|\partial_{t} b_{1}(t)\right|+\left|\partial_{t t} b_{1}(t)\right|+\left|\partial_{t t} a(t)\right| \leq C e^{2 t}
$$

and,

$$
\left|\partial_{\theta_{j}} b_{1}\right|+\left|\partial_{\theta_{j}, \theta_{k}} b_{1}\right|+\partial_{t, \theta_{j}} b_{1}|+| \partial_{t, \theta_{j}, \theta_{k}} b_{1} \mid \leq C e^{2 t}
$$

But,

$$
\Delta_{\theta}=\Delta_{g_{y_{i}, e^{t}, \mathbb{S}_{3}}}=-\frac{\partial_{\theta^{l}}\left[\tilde{g}^{\theta^{l} \theta^{j}}\left(e^{t}, \theta\right) \sqrt{\left|\tilde{g}^{k}\left(e^{t}, \theta\right)\right|} \partial_{\theta^{j}}\right]}{\sqrt{\left|\tilde{g}^{k}\left(e^{t}, \theta\right)\right|}} .
$$

Then,

$$
A_{i}:=\left[\left[\frac{\partial_{\theta^{l}}\left(\tilde{g}^{\theta^{l} \theta^{j}} \sqrt{\left|\tilde{g}^{k}\right|} \partial_{\theta^{j}}\right)}{\sqrt{\left|\tilde{g}^{k}\right|}}\right]^{\xi_{i}}-\left[\frac{\partial_{\theta^{l}}\left(\tilde{g}^{\theta^{l} \theta^{j}} \sqrt{\left|\tilde{g}^{k}\right|} \partial_{\theta^{j}}\right)}{\sqrt{\left|\tilde{g}^{k}\right|}}\right]\right]\left(\tilde{w}_{i}^{\xi_{i}}\right)=B_{i}+D_{i}
$$

where,

$$
B_{i}=\left[\tilde{g}^{\theta^{l} \theta^{j}}\left(e^{t^{\xi_{i}}}, \theta\right)-\tilde{g}^{\theta^{l} \theta^{j}}\left(e^{t}, \theta\right)\right] \partial_{\theta^{l} \theta^{j}} \tilde{w}_{i}^{\xi_{i}}
$$

and,

$$
D_{i}=\left[\frac{\partial_{\theta^{l}}\left[\tilde{g}^{\theta^{l} \theta^{j}}\left(e^{t^{\xi_{i}}}, \theta\right) \sqrt{\left|\tilde{g}^{k}\right|}\left(e^{t^{\xi_{i}}}, \theta\right)\right]}{\sqrt{\left|\tilde{g}^{k}\right|}\left(e^{\xi_{i}}, \theta\right)}-\frac{\partial_{\theta^{l}}\left[\tilde{g}^{\theta^{l} \theta^{j}}\left(e^{t}, \theta\right) \sqrt{\left|\tilde{g}^{k}\right|}\left(e^{t}, \theta\right)\right]}{\sqrt{\left|\tilde{g}^{k}\right|}\left(e^{t}, \theta\right)}\right] \partial_{\theta^{j}} \tilde{w}_{i}^{\xi_{i}}
$$

Clearly, we can choose $\epsilon_{1}>0$ such that:

$$
\left|\partial_{r} \tilde{g}_{i j}^{k}(x, r, \theta)\right|+\left|\partial_{r} \partial_{\theta^{m}} \tilde{g}_{i j}^{k}(x, r, \theta)\right| \leq C r, x \in B\left(x_{0}, \epsilon_{1}\right) r \in\left[0, \epsilon_{1}\right], \quad \theta \in U^{k}
$$

finally, 


$$
A_{i} \leq C_{k}\left|e^{2 t}-e^{2 t^{\xi_{i}}}\right|\left[\left|\nabla_{\theta} \tilde{w}_{i}^{\xi_{i}}\right|+\left|\nabla_{\theta}^{2}\left(\tilde{w}_{i}^{\xi_{i}}\right)\right|\right]
$$

We take, $C=\max \left\{C_{i}, 1 \leq i \leq q\right\}$ and we use (14). Proposition 2.6 is proved.

We have,

$$
\begin{gathered}
c\left(y_{i}, t, \theta\right)=1+\partial_{t} a+h e^{2 t}, \\
b_{2}(t, \theta)=\partial_{t t}\left(\sqrt{b_{1}}\right)=\frac{1}{2 \sqrt{b_{1}}} \partial_{t t} b_{1}-\frac{1}{4\left(b_{1}\right)^{3 / 2}}\left(\partial_{t} b_{1}\right)^{2}, \\
c_{2}=\left[\frac{1}{\sqrt{b_{1}}} \Delta_{\theta}\left(\sqrt{b_{1}}\right)+\left|\nabla_{\theta} \log \left(\sqrt{b_{1}}\right)\right|^{2}\right],
\end{gathered}
$$

We assume that $\lambda \leq \lambda_{i}+2=-\log u_{i}\left(y_{i}\right)+2$, which will be choosen later.

We work on $\left[\lambda, t_{i}\right] \times \mathbb{S}_{3}$ with $t_{i}=\log l_{i} \rightarrow-\infty, l_{i}$ as in the proposition 1 . For $i$ large $\log l_{i}>>$ $\lambda_{i}+2$.

The functions $v_{i}$ tend to a radially symmetric function, then, $\partial_{\theta_{j}} w_{i}^{\lambda} \rightarrow 0$ if $i \rightarrow+\infty$ and,

$$
\frac{\partial_{\theta_{j}} w_{i}^{\lambda}(t, \theta)}{w_{i}^{\lambda}}=\frac{e^{(n-2)\left[\left(\lambda-\lambda_{i}\right)+\left(\xi_{i}-t\right)\right] / 2} e^{\left[\left(\lambda-\lambda_{i}\right)+\left(\xi_{i}-t\right)\right]}\left(\partial_{\theta_{j}} v_{i}\right)\left(e^{\left[\left(\lambda-\lambda_{i}\right)+(\lambda-t)\right]} \theta\right)}{e^{(n-2)\left[\left(\lambda-\lambda_{i}\right)+(\lambda-t)\right] / 2} v_{i}\left[e^{\left(\lambda-\lambda_{i}\right)+(\lambda-t)} \theta\right]} \leq \bar{C}_{i},
$$

where $\bar{C}_{i}$ does not depend on $\lambda$ and tends to 0 . We have also,

$$
\left|\partial_{\theta} w_{i}^{\lambda}(t, \theta)\right|+\left|\partial_{\theta, \theta} w_{i}^{\lambda}(t, \theta)\right| \leq \tilde{C}_{i} w_{i}^{\lambda}(t, \theta), \tilde{C}_{i} \rightarrow 0
$$

and,

$$
\left|\partial_{\theta} \tilde{w}_{i}^{\lambda}(t, \theta)\right|+\left|\partial_{\theta, \theta} \tilde{w}_{i}^{\lambda}(t, \theta)\right| \leq \tilde{C}_{i} \tilde{w}_{i}^{\lambda}(t, \theta), \tilde{C}_{i} \rightarrow 0
$$

$\tilde{C}_{i}$ does not depend on $\lambda$.

Now, we set:

$$
\bar{w}_{i}=\tilde{w}_{i}-\frac{\tilde{m}_{i}}{2} e^{2 t}
$$


with, $m_{i}=\frac{1}{2} u_{i}\left(x_{i}\right)^{1 / 3} \min _{M} u_{i}$. As in [2], we have,

Lemma 2.7. There is $\nu<0$ such that for $\lambda \leq \nu$ :

$$
\bar{w}_{i}^{\lambda}(t, \theta)-\bar{w}_{i}(t, \theta) \leq 0, \forall(t, \theta) \in\left[\lambda, t_{i}\right] \times \mathbb{S}_{3} .
$$

Let $\xi_{i}$ be the following real number,

$$
\xi_{i}=\sup \left\{\lambda \leq \lambda_{i}+2, \bar{w}_{i}^{\xi_{i}}(t, \theta)-\bar{w}_{i}(t, \theta) \leq 0, \forall(t, \theta) \in\left[\xi_{i}, t_{i}\right] \times \mathbb{S}_{3}\right\}
$$

Like in [2], we use the previous lemma to show:

$$
\bar{w}_{i}^{\xi_{i}}-\bar{w}_{i} \leq 0 \Rightarrow \bar{Z}_{i}\left(\bar{w}_{i}^{\xi_{i}}-\bar{w}_{i}\right) \leq 0 \text {. }
$$

We have,

$$
\begin{gathered}
\bar{Z}_{i}\left(\tilde{w}_{i}^{\xi_{i}}-\tilde{w}_{i}\right) \leq 8 b_{1}^{\xi_{i}}\left[\left(\tilde{w}_{i}^{\xi_{i}}\right)^{3}-\tilde{w}_{i}^{3}\right]+O(1)\left(e^{2 t}-e^{2 t \xi_{i}}\right)+O(1) \tilde{w}_{i}^{\xi_{i}}\left(e^{2 t}-e^{2 t \xi_{i}}\right) . \\
-\bar{Z}_{i}\left(e^{2 t^{\xi_{i}}}-e^{2 t}\right)=\left(4-1-\partial_{t} a-h e^{2 t}+b_{1}^{-1 / 2} b_{2}-c_{2}\right)\left(e^{2 t \xi_{i}}-e^{2 t}\right) \leq c_{3}\left(e^{2 t^{\xi_{i}}}-e^{2 t}\right)
\end{gathered}
$$

Thus,

$$
\bar{Z}_{i}\left(\bar{w}_{i}^{\xi_{i}}-\bar{w}_{i}\right) \leq 8 b_{1}^{\xi_{i}}\left[\left(\tilde{w}_{i}^{\xi_{i}}\right)^{3}-\tilde{w}_{i}^{3}\right]+\left(c_{3} m_{i}-c_{4}\right)\left(e^{2 t^{\xi_{i}}}-e^{2 t}\right) .
$$

with, $c_{3}, c_{4}>0$.

But,

$$
0<\tilde{w}_{i}^{\xi_{i}} \leq 2 e, \quad \tilde{w}_{i} \geq \frac{m_{i}}{2} e^{2 t} \text { and } \tilde{w}_{i}^{\xi_{i}}-\tilde{w}_{i} \leq \frac{m_{i}}{2}\left(e^{2 t \xi_{i}}-e^{2 t}\right)
$$

and,

$\left(\tilde{w}_{i}^{\xi_{i}}\right)^{3}-\tilde{w}_{i}^{3}=\left(\tilde{w}_{i}^{\xi_{i}}-\tilde{w}_{i}\right)\left[\left(\tilde{w}_{i}^{\xi_{i}}\right)^{2}+\tilde{w}_{i}^{\xi_{i}} \tilde{w}_{i}+\tilde{w}_{i}^{2}\right] \leq\left(\tilde{w}_{i}^{\xi_{i}}-\tilde{w}_{i}\right)\left(\tilde{w}_{i}^{\xi_{i}}\right)^{2}+\left(\tilde{w}_{i}^{\xi_{i}}-\tilde{w}_{i}\right) \frac{m^{2} e^{2 t}}{4}+\left(\tilde{w}_{i}^{\xi_{i}}-\tilde{w}_{i}\right) \frac{m}{2} e^{t} \tilde{w}_{i}^{\xi_{i}}$ 
then,

$$
\bar{Z}_{i}\left(\bar{w}_{i}^{\xi_{i}}-\bar{w}_{i}\right) \leq\left[\left[\frac{a m_{i}^{3}}{16}-O(1)\right]+\left[\frac{a m_{i}^{2}}{8}-O(1)\right] e^{t} \tilde{w}_{i}^{\xi_{i}}\right]\left(e^{2 t^{\xi_{i}}}-e^{2 t}\right) \leq 0 .
$$

If we use the maximum principle and the Hopf lemma, we obtain (as in [2]):

$$
\max _{\theta \in \mathbb{S}_{3}} w_{i}\left(t_{i}, \theta\right) \leq \min _{\theta \in \mathbb{S}_{3}} w_{i}\left(2 \xi_{i}-t_{i}, \theta\right)
$$

we can write (using proposition 2):

$$
l_{i}\left[u_{i}\left(y_{i}\right)\right]^{1 / 3} \min _{M} u_{i} \leq c,
$$

\section{Metric AND LAPLACiAn}

In this section, we give some remarks on Polar Geodesic coordinates and the Laplacian in these coordinates. First by using the Jacobi Fields we can have an expansion of the metric in geodesic coordinates, we can extend this result to polar geodesic coordinates.

\section{Estimate of the metric in Polar Coordinates.}

Let us consider a riemannian manifold (not necessarily compact) $(M, g)$. We set $g_{x, i j}$ the components of the metric in the exponential chart centered at $x$.

By the Gauss formula we have:

$$
g=d s^{2}=d t^{2}+g_{i j}^{k}(r, \theta) d \theta^{i} d \theta^{j}=d t^{2}+r^{2} \tilde{g}_{i j}^{k}(r, \theta) d \theta^{i} d \theta^{j}=g_{x, i j} d x^{i} d x^{j},
$$

in polar chart centered at $x,] 0, \epsilon_{0}\left[\times U^{k}\right.$, with $\left(U^{k}, \psi\right)$ a chart of the unit sphere $\mathbb{S}_{n-1}$.

We can write the volume lement as:

$$
d V_{g}=r^{n-1} \sqrt{\left|\tilde{g}^{k}\right|} d r d \theta^{1} \ldots d \theta^{n-1}=\sqrt{\left[\operatorname{det}\left(g_{x, i j}\right)\right]} d x^{1} \ldots d x^{n},
$$

thus,

$$
d V_{g}=r^{n-1} \sqrt{\left[\operatorname{det}\left(g_{x, i j}\right)\right]}\left[\exp _{x}(r \theta)\right] \alpha^{k}(\theta) d r d \theta^{1} \ldots d \theta^{n-1}
$$


with, $\alpha^{k}$ is such that $d \sigma_{\mathbb{S}_{n-1}}=\alpha^{k}(\theta) d \theta^{1} \ldots d \theta^{n-1}$. (volume element of the sphere in the chart $\left.\left(U^{k}, \psi\right)\right)$.

Thus,

$$
\sqrt{\left|\tilde{g}^{k}\right|}=\alpha^{k}(\theta) \sqrt{\left[\operatorname{det}\left(g_{x, i j}\right)\right]}
$$

Proposition: Let us consider $x_{0} \in M$, there is $\epsilon_{1}>0$ and $U^{k}$, such that:

$$
\left|\partial_{r} \tilde{g}_{i j}^{k}(x, r, \theta)\right|+\left|\partial_{r} \partial_{\theta^{m}} \tilde{g}_{i j}^{k}(x, r, \theta)\right| \leq C r, \forall x \in B\left(x_{0}, \epsilon_{1}\right) \forall r \in\left[0, \epsilon_{1}\right], \forall \theta \in U^{k}
$$

and,

$$
\left|\partial_{r}\right| \tilde{g}^{k}|(x, r, \theta)|+\partial_{r} \partial_{\theta^{m}}\left|\tilde{g}^{k}\right|(x, r, \theta) \leq C r, \forall x \in B\left(x_{0}, \epsilon_{1}\right) \forall r \in\left[0, \epsilon_{1}\right], \forall \theta \in U^{k}
$$

and,

$\left|\partial_{r} \partial_{\theta_{m}}\left[\frac{\sqrt{\left|\tilde{g}^{k}\right|}}{\alpha^{k}(\theta)}\right](x, r, \theta)\right|+\left|\partial_{r} \partial_{\theta_{m}} \partial_{\theta_{m^{\prime}}}\left[\frac{\sqrt{\left|\tilde{g}^{k}\right|}}{\alpha^{k}(\theta)}\right](x, r, \theta)\right| \leq C r \forall(x, r, \theta) \in B\left(x_{0}, \epsilon_{1}\right) \times\left[0, \epsilon_{1}\right] \times U^{k}$

\section{Proof:}

Next, we use Einstein convention:

First, we consider a chart $(\Omega, \varphi)$ in $x_{0}$, such that $\bar{\Omega}$ is compact, we can assume it normal at $x_{0}$.

According to lemma 2.3.7 of [He], for all $x_{0} \in M$ there is $\epsilon_{0}>0$ such that the application $u:(x, v) \rightarrow \exp _{x}(v)$ on $B\left(x_{0}, \epsilon_{0}\right) \times B\left(0, \epsilon_{0}\right)$ in $M$ is $C^{\infty}$ and for all $x \in B\left(x_{0}, \epsilon\right)$ the application $v \rightarrow \exp _{x}(v)$ is a diifeomorphism of $B\left(0, \epsilon_{0}\right)$ in $B\left(x_{0}, \epsilon_{0}\right)$ with $\exp _{x}[\partial B(0, \mu)]=\partial B(x, \mu)$, $\mu \leq \epsilon_{0}$. Without loss of generality we can assume that $B\left(x, \epsilon_{0}\right) \subset \subset \Omega$ for all $x \in B\left(x_{0}, \epsilon_{0}\right)$.

Thus, we have for all $x \in B\left(x_{0}, \epsilon_{0}\right),\left[B\left(x, \epsilon_{0}\right), \exp _{x}^{-1}\right]$ is a normal chart in $x$.(In this case we can define polar coordinates). For all $x \in B\left(x_{0}, \epsilon_{0}\right)$ :

$$
g_{x, i j}(z)=g(z)\left(\partial_{z^{i}, x}, \partial_{z^{j}, x}\right),
$$

with $\partial_{z^{i}, x}$ is the canonical vector field in the exponential chart. 
We set $a_{i}^{k}(z, x)=\frac{\left(\varphi o \exp _{x}\right)^{k}}{\partial z^{i}}\left[\exp _{x}^{-1}(z)\right]$, then $\partial_{z^{i}, x}=a_{i}^{k}(z, x) \partial_{u^{k}, \varphi}$,

with $\partial_{u^{k}, \varphi}$, the canonical vector field with respect to chart $(\Omega, \varphi)$, this vector field do not depends on $x$ and the functions $a_{i}^{k}$ are regular of $z$ and $x$. We obtain,

$$
g_{x, i j}(z)=g(z)\left[a_{i}^{k}(z, x) \partial_{u^{k}, \varphi} ; a_{j}^{l}(z, x) \partial_{u^{l}, \varphi}\right]=a_{i}^{k}(z, x) a_{j}^{l}(z, x) g_{k l}(z),
$$

with $g_{k l}$ the component of $g$ in the chart $(\Omega, \varphi)$.

We have $z=\exp _{x}(y), y \in B\left(0, \epsilon_{0}\right) \subset \mathbb{R}^{n}$ and $y=r \theta$ in polar coordinates, thus $(x, r, \theta) \rightarrow$ $g_{x, i j}\left[\exp _{x}(r \theta)\right]$ is $C^{\infty}$ of $x, r$ et $\theta$.

We have, by definition, $g_{i j}^{k}(r \theta)=g_{\left[\exp _{x}(r \theta)\right]}\left(\partial_{\theta^{i}, x}, \partial_{\theta^{j}, x}\right)$, (canonical vector fields).

We can write,

$$
\partial_{\theta^{i}, x}=r b_{i}^{k}(\theta) \partial_{z^{k}, x}
$$

with $b_{i}^{j}$ regular. (Note, that here, we can use the function $\theta^{i}$, as regular function in the chart of the sphere. With this procedure we can deduce the component of the metric in polar coordinates ( a good expansion)). Thus,

$$
g_{i j}^{k}(r, \theta)=r^{2} g\left[\exp _{x}(r \theta)\right]\left(b_{i}^{k} \partial_{z^{k}, x}, b_{j}^{l} \partial_{z^{l}, x}\right),
$$

Then,

$$
g_{i j}^{k}(r, \theta)=r^{2} b_{i}^{k}(\theta) b_{j}^{l}(\theta) g_{x, k l}\left[\exp _{x}(r \theta)\right] .
$$

Thus, the functions $\tilde{g}_{i j}^{k}:(x, r, \theta) \rightarrow b_{j}^{k} b_{j}^{l} g_{x, k l}\left[\exp _{x}(r \theta)\right]$ is regular of $x, r$ and $\theta$. We have,

$$
\partial_{r} \tilde{g}_{i j}^{k}(x, 0, \theta)=b_{i}^{k}(\theta) b_{j}^{k}(\theta) c^{m}(\theta) \partial_{m} g_{x, k l}(x)=0,
$$

because the exponential chart is normal in $x$ and $g_{x, k l}$ are the component of $g$ in this chart. We have:

$$
\partial_{\theta^{m}} \tilde{g}_{i j}^{k}(x, r, \theta)=\tilde{b}_{i}^{k}(\theta) \tilde{b}_{j}^{l}(\theta) g_{x, k l}\left[\exp _{x}(r \theta)\right]+r \bar{b}_{i}^{k}(\theta) \bar{b}_{j}^{l}(\theta) \bar{c}_{m}^{s}(\theta) \partial_{s} g_{x, k l}\left[\exp _{x}(r \theta)\right],
$$

We also have: 


$$
\begin{gathered}
\partial_{r} \partial_{\theta^{m}} \tilde{g}_{i j}^{k}(x, r, \theta)=u_{i j m r}^{k l q}(\theta) \partial_{q} g_{x, k l}\left[\exp _{x}(r \theta)\right]+v_{i j m r}^{k l}(\theta) w^{s}(t) \partial_{s} g_{x, k l}\left[\exp _{x}(r \theta)\right]+ \\
+r h_{i j r m}^{k l s t}(\theta) \partial_{s t} g_{x, k l}\left[\exp _{x}(r \theta)\right]
\end{gathered}
$$

Thus,

$$
\partial_{r} \partial_{\theta^{m}} \tilde{g}_{i j}^{k}(x, 0, \theta)=0, \forall, x \in B\left(x_{0}, \epsilon_{0}\right), \forall \theta \in U^{k}
$$

Thus, we obtain:

$$
\partial_{r} \tilde{g}_{i j}^{k}(x, 0, \theta)=\partial_{r} \partial_{\theta^{m}} \tilde{g}_{i j}^{k}(x, 0, \theta)=0, \forall x \in B\left(x_{0}, \epsilon_{0}\right), \forall \theta \in U^{k}
$$

Because $\sqrt{\left|\tilde{g}^{k}\right|}=\alpha^{k}(\theta) \sqrt{\left[\operatorname{det}\left(g_{x, i j}\right)\right]}$, we deduce,

$$
\partial_{r}\left(\log \sqrt{\left|\tilde{g}^{k}\right|}\right)=\partial_{r}\left[\log \left(\sqrt{\left[\operatorname{det}\left(g_{x, i j}\right)\right]}\right)\right] .
$$

We use the definition of the determinant

$$
\operatorname{det}\left[g_{x, i j}\right]\left[\exp _{x}(r \theta)\right]=\Sigma \Pi g_{x, k l}\left[\exp _{x}(r \theta)\right]
$$

Thus,

$$
\partial_{r} \operatorname{det}\left[g_{x, i j}\right](x)=\Sigma \Pi\left[g_{x, k l}(x)\right] a^{s}(\theta) \partial_{s} g_{x, m n}(x)=0,
$$

because the exponential chart is normal at $x$.

Finaly

$$
\partial_{r}\left|\tilde{g}^{k}\right|(x, 0, \theta)=0, \forall x \in B\left(x_{0}, \epsilon_{0}\right), \forall \theta \in U^{k} .
$$

We also have $\partial_{r} \partial_{\theta^{m}} \tilde{g}_{i j}^{k}(x, 0, \theta)$, to prove that,

$$
\partial_{r} \partial_{\theta^{m}}\left|\tilde{g}^{k}\right|(x, 0, \theta)=0 .
$$

If we set $D_{m}=\partial_{\theta_{m}} \frac{\sqrt{\left|\tilde{g}^{k}\right|}}{\alpha^{k}(\theta)}(x, r, \theta)$, 


$$
D_{m}=r \sum \Pi \beta_{m}^{l}(\theta) \partial_{l} g_{x, i j}\left[\exp _{x}(r \theta)\right] g_{x, i j}\left[\exp _{x}(r \theta)\right]
$$

We have $D_{m}(x, 0, \theta)=0$ and,

$$
\partial_{r} D_{m}(x, 0, \theta)=\lim _{r \rightarrow 0}\left(D_{m} / r\right)(x, r, \theta)=0
$$

Thus

$$
\partial_{r} \partial_{\theta_{m}}\left[\frac{\sqrt{\left|\tilde{g}^{k}\right|}}{\alpha^{k}(\theta)}\right](x, 0, \theta)=0
$$

$\partial_{\theta_{m^{\prime}}} D_{m}=r \Sigma \Pi \partial_{m^{\prime}} \beta_{m}^{l} \partial_{l} g_{x, i j} g_{x, i j}+r^{2} \Sigma \Pi \beta_{m}^{l} \beta_{m^{\prime}}^{l^{\prime}} \partial_{l l^{\prime}} g_{x, i j} g_{x, i j}+r^{2} \Sigma \Pi \beta_{m}^{l} \beta_{m^{\prime}}^{l^{\prime}} \partial_{l} g_{x, i j} \partial_{l^{\prime}} g_{x, i j} g_{x, i j}$, but, $\partial_{\theta_{m^{\prime}}} D_{m}(x, 0, \theta)=0$, we have,

$$
\partial_{r} \partial_{\theta_{m^{\prime}}} D_{m}(x, 0, \theta)=\lim _{r \rightarrow 0}\left[\partial_{\theta_{m^{\prime}}} D_{m} / r\right]=0
$$

Thus,

$$
\partial_{r} \partial_{\theta_{m}} \partial_{\theta m^{\prime}}\left[\frac{\sqrt{\left|\tilde{g}^{k}\right|}}{\alpha^{k}(\theta)}\right](x, 0, \theta)=0
$$

Finaly

$\partial_{r} \tilde{g}_{i j}^{k}(x, 0, \theta)=\partial_{r} \partial_{\theta^{m}} \tilde{g}_{i j}^{k}(x, 0, \theta)=0 \forall x \in B\left(x_{0}, \epsilon_{0}\right), \forall \theta \in U^{k}$

$\partial_{r}\left|\tilde{g}^{k}\right|(x, 0, \theta)=\partial_{r} \partial_{\theta^{m}}\left|\tilde{g}^{k}\right|(x, 0, \theta)=0 \forall x \in B\left(x_{0}, \epsilon_{0}\right), \forall \theta \in U^{k}$

$\partial_{r} \partial_{\theta_{m}}\left[\frac{\sqrt{\left|\tilde{g}^{k}\right|}}{\alpha^{k}(\theta)}\right](x, 0, \theta)=\partial_{r} \partial_{\theta_{m}} \partial_{\theta m^{\prime}}\left[\frac{\sqrt{\left|\tilde{g}^{k}\right|}}{\alpha^{k}(\theta)}\right](x, 0, \theta)=0 \forall x \in B\left(x_{0}, \epsilon_{0}\right), \forall \theta \in U^{k} .(* * * *)$

We can reduce the open set $U^{k}$ to have these estimates and use the uniform continuity to have the estimates. 


\section{The Laplacian in polar coordinates}

We can write in $\left[0, \epsilon_{1}\right] \times U^{k}$,

$$
-\Delta=\partial_{r r}+\frac{n-1}{r} \partial_{r}+\partial_{r}\left[\log \sqrt{\left|\tilde{g}^{k}\right|} \partial_{r}+\frac{1}{r^{2} \sqrt{\left|\tilde{g}^{k}\right|}} \partial_{\theta^{i}}\left(\tilde{g}^{\theta^{i} \theta^{j}} \sqrt{\left|\tilde{g}^{k}\right|} \partial_{\theta^{j}}\right) .\right.
$$

On a,

$$
-\Delta=\partial_{r r}+\frac{n-1}{r} \partial_{r}+\partial_{r} \log J(x, r, \theta) \partial_{r}+\frac{1}{r^{2} \sqrt{\left|\tilde{g}^{k}\right|}} \partial_{\theta^{i}}\left(\tilde{g}^{\theta^{i} \theta^{j}} \sqrt{\left|\tilde{g}^{k}\right|} \partial_{\theta^{j}}\right) .
$$

We can write the Laplacian (radial and angular decomposition, see S. Lang book),

$$
-\Delta=\partial_{r r}+\frac{n-1}{r} \partial_{r}+\partial_{r}[\log J(x, r, \theta)] \partial_{r}-\Delta_{\mathbb{S}_{r}(x)},
$$

with $\Delta_{\mathbb{S}_{r}(x)}$ the Laplacian on $\mathbb{S}_{r}(x)$.

Locally we can write $\Delta_{\mathbb{S}_{r}(x)}$ as:

$$
-\Delta_{\mathbb{S}_{r}(x)}=\frac{1}{r^{2} \sqrt{\left|\tilde{g}^{k}\right|}} \partial_{\theta^{i}}\left(\tilde{g}^{\theta^{i} \theta^{j}} \sqrt{\left|\tilde{g}^{k}\right|} \partial_{\theta^{j}}\right)
$$

We set: $L_{\theta}(x, r)(\ldots)=r^{2} \Delta_{\mathbb{S}_{r}(x)}(\ldots)\left[\exp _{x}(r \theta)\right]$.

The operator $L_{\theta}(x, r)$ act on $C^{2}\left(\mathbb{S}_{n-1}\right)$ functions globaly and not depends on the chart on $\mathbb{S}_{n-1}$ and locally we can write it as:

$$
L_{\theta}(x, r)=-\frac{1}{\sqrt{\left|\tilde{g}^{k}\right|}} \partial_{\theta^{i}}\left[\tilde{g}^{\theta^{i} \theta^{j}} \sqrt{\left|\tilde{g}^{k}\right|} \partial_{\theta^{j}}\right]
$$

We have

$$
\Delta=\partial_{r r}+\frac{n-1}{r} \partial_{r}+\partial_{r}[J(x, r, \theta)] \partial_{r}-\frac{1}{r^{2}} L_{\theta}(x, r) .
$$

We set, for $u$ a function on $M, \bar{u}(r, \theta)=u o \exp _{x}(r \theta)$ in polar coordnates centered in $x$ : 


$$
\begin{gathered}
-\Delta u=\partial_{r r} \bar{u}+\frac{n-1}{r} \partial_{r} \bar{u}+\partial_{r}[J(x, r, \theta)] \partial_{r} \bar{u}-\Delta_{\mathbb{S}_{r}(x)}\left(u_{\mid \mathbb{S}_{r}(x)}\right)\left[\exp _{x}(r \theta)\right], \\
r^{2} \Delta_{\mathbb{S}_{r}(x)}\left(u_{\mid \mathbb{S}_{r}(x)}\right)\left[\exp _{x}(r \theta)\right]=-\frac{1}{\sqrt{\left|\tilde{g}^{k}\right|}} \partial_{\theta^{i}}\left[\tilde{g}^{\theta^{i} \theta^{j}} \sqrt{\left|\tilde{g}^{k}\right|} \partial_{\theta^{j}}\left[u o \exp _{x}(r \theta)\right]\right]=L_{\theta}(x, r) \bar{u} .
\end{gathered}
$$

Thus,

$$
-\Delta u=\partial_{r r} \bar{u}+\frac{n-1}{r} \partial_{r} \bar{u}+\partial_{r}[J(x, r, \theta)] \partial_{r} \bar{u}-\frac{1}{r^{2}} L_{\theta}(x, r) \bar{u} .
$$

\section{Lemma:}

The operator $L_{\theta}(x, r)$ is a Laplacian on $\mathbb{S}_{n-1}$ for a particular metric which depends on $r$.

\section{Proof on the Lemma:}

We have $\Delta_{\mathbb{S}_{r}(x)}=\Delta_{i_{x, r}^{*}(g)}$ with $i_{x, r}$ the identity map from $\mathbb{S}_{r}(x)$ in $M$ and $i_{x, r}^{*}(g)=\tilde{g}$ the induced metric on the submanifold $\mathbb{S}_{r}(x)$ of $M$.

The map $\exp _{x}$ induce a diffeomorphism from $\mathbb{S}_{r}(x)$ into $\mathbb{S}_{n-1}^{r}$, we have:

$$
\Delta_{\mathbb{S}_{r}(x)} u_{\mid \mathbb{S}_{r}(x)}=\Delta_{i_{x, r}^{*}(g)} u_{\mid \mathbb{S}_{r}(x)}=\Delta_{\tilde{g}} u_{\mid \mathbb{S}_{r}(x)}=\Delta_{\exp _{x}^{*}(\tilde{g}), \mathbb{S}_{n-1}^{r}} u o \exp _{x}(v),
$$

Let us consider $\tilde{z}$ the map from $\mathbb{S}_{n-1}$ into $\mathbb{S}_{n-1}^{r}$ defined by $\theta \rightarrow r \theta$. Thus,

$$
\Delta_{\exp _{x}^{*}(\tilde{g})} u o \exp _{x}(v)=\Delta_{\tilde{z}^{*}\left[\exp _{x}^{*}(\tilde{g})\right]} u o \exp _{x}(r \theta)
$$

For a chart $\left(\psi, U^{k}\right)$ on $\mathbb{S}_{n-1}$, the polar chart in $x$ is, $\left(\varphi_{0},\left[0, \epsilon_{0}\right] \times U^{k}\right)$, where $\varphi_{0}=\exp _{x} o \tilde{z} o \psi^{-1}$. The $g_{j l}^{k}$ are, by definition:

$$
g_{j l}^{k}(r, \theta)=g_{\exp _{x}(r \theta)}\left(\partial_{j, \varphi_{0}}, \partial_{l, \varphi_{0}}\right),
$$

with, $\partial_{j, \varphi_{0}}, \partial_{l, \varphi_{0}}$ the canonical vector fields for the chart $\left(\varphi_{0},\left[0, \epsilon_{0}\right] \times U^{k}\right)$.

By definition, take $h$ a function defined in a neighborhood of $x$ :

$$
\partial_{j, \varphi_{0}}(h)=\frac{\partial\left(h o \varphi_{0}\right)}{\partial \bar{\theta}_{j}}=\frac{\partial\left(h o \exp _{x} o \tilde{z} o \psi^{-1}\right)}{\partial \bar{\theta}_{j}}
$$


with $\psi(\theta)=\left(\bar{\theta}_{1}, \ldots, \bar{\theta}_{n-1}\right)$ and $\theta_{0}=r$ (angular and radial derivations).

We look to the angular components, $\partial_{j, \varphi_{0}}$ with $1 \leq j \leq n-1, j \in \mathbb{N}$, thus,

$$
\begin{gathered}
\partial_{j, \varphi_{0}}(h)=\frac{\partial\left(h o i_{x, r} o \exp _{x} o \tilde{z} o \psi^{-1}\right)}{\partial \bar{\theta}_{j}}, \\
\frac{\partial\left(h o \varphi_{0}\right)}{\partial \bar{\theta}_{j}}=\frac{\partial\left(h o i_{x, r} o \varphi_{0}\right)}{\partial \bar{\theta}_{j}}
\end{gathered}
$$

Thus, if write $\bar{\partial}_{j}, \bar{\partial}_{l}$ as a canonical vector fields of the unit sphre in the chart $\left(\psi, U^{k}\right)$, we obtain, we use $d$ for the differential,

$$
\partial_{j, \varphi_{0}}=d\left(i_{x, r} o \exp _{x} o \tilde{z}\right)\left(\bar{\partial}_{j}\right)
$$

Thus,

$$
g_{j l}^{k}(r, \theta)=g_{\exp _{x}(r \theta)}\left[d \left(i_{x, r} o \exp _{x} o \tilde{z}\left(\bar{\partial}_{j}\right), d\left(i_{x, r} o \exp _{x} o \tilde{z}\left(\bar{\partial}_{l}\right)\right],\right.\right.
$$

we use the definition of the pull-back:

$$
g_{j l}^{k}(r, \theta)=\tilde{z}^{*}\left[\exp _{x}^{*}\left[i_{x, r}^{*}(g)\right]\right]_{\theta}\left(\bar{\partial}_{j}, \bar{\partial}_{l}\right)=\tilde{z}^{*}\left[\exp _{x}^{*}(\tilde{g})\right]\left(\bar{\partial}_{j}, \bar{\partial}_{l}\right),
$$

Thus we have the component $j, l$ of the mteric $\tilde{z}^{*}\left[\exp _{x}^{*}(\tilde{g})\right]$ on the unit sphere $\mathbb{S}_{n-1}$.

Finaly we have locally and globaly:

$$
-\Delta_{\tilde{z}^{*}\left[\exp _{x}^{*}(\tilde{g})\right], \mathbb{S}_{n-1}} u o \exp _{x}(r \theta)=\frac{1}{r^{2} \sqrt{\left|\tilde{g}^{k}\right|}} \partial_{\theta^{i}}\left[\tilde{g}^{\theta^{i} \theta^{j}} \sqrt{\left|\tilde{g}^{k}\right|} \partial_{\theta^{j}} u o \exp _{x}\left(r \psi^{-1}\right)\right]
$$

Now, if we consider the metric on $\mathbb{S}_{n-1}$ defined by,

$$
g_{x, r, \mathbb{S}_{n-1}}=r^{-2} \tilde{z}^{*}\left[\exp _{x}^{*}(\tilde{g})\right]
$$

this metric is well-defined and is such that:

$$
g_{x, r, \mathbb{S}_{n-1} j l}=r^{-2} g_{j l}^{k}=\tilde{g}_{j l}^{k},
$$

Bibliography for this section: 
[Au] T. Aubin. Some Nonlinear Problems in Riemannian Geometry. Springer-Verlag 1998.

[He] E. Hebey, Analyse non lineaire sur les Variétés, Editions Diderot.

[L] S. Lang. Differentiable and Riemannian manifolds.

\section{REFERENCES}

[1] T. Aubin. Some Nonlinear Problems in Riemannian Geometry. Springer-Verlag 1998

[2] S.S Bahoura. Majorations du type $\sup u \times \inf u \leq c$ pour l'équation de la courbure scalaire sur un ouvert de $\mathbb{R}^{n}, n \geq 3$. J. Math. Pures. Appl.(9) 832004 no, 9, 1109-1150.

[3] S.S. Bahoura. Harnack inequalities for Yamabe type equations. Bull. Sci. Math. 133 (2009), no. 8, 875-892

[4] S.S. Bahoura. Lower bounds for sup+inf and sup $\times$ inf and an extension of Chen-Lin result in dimension 3 . Acta Math. Sci. Ser. B Engl. Ed. 28 (2008), no. 4, 749-758

[5] S.S. Bahoura. Estimations uniformes pour l'equation de Yamabe en dimensions 5 et 6. J. Funct. Anal. 242 (2007), no. 2, 550-562.

[6] S.S. Bahoura. sup $\times$ inf inequality on manifold of dimension 3, to appear in MATHEMATICA AETERNA

[7] H. Brezis, YY. Li. Some nonlinear elliptic equations have only constant solutions. J. Partial Differential Equations 19 (2006), no. 3, 208-217.

[8] H. Brezis, YY. Li , I. Shafrir. A sup+inf inequality for some nonlinear elliptic equations involving exponential nonlinearities. J.Funct.Anal.115 (1993) 344-358.

[9] H.Brezis and F.Merle, Uniform estimates and blow-up bihavior for solutions of $-\Delta u=V e^{u}$ in two dimensions, Commun Partial Differential Equations 16 (1991), 1223-1253.

[10] L. Caffarelli, B. Gidas, J. Spruck. Asymptotic symmetry and local behavior of semilinear elliptic equations with critical Sobolev growth. Comm. Pure Appl. Math. 37 (1984) 369-402.

[11] C-C.Chen, C-S. Lin. Estimates of the conformal scalar curvature equation via the method of moving planes. Comm. Pure Appl. Math. L(1997) 0971-1017.

[12] C-C.Chen, C-S. Lin. A sharp sup+inf inequality for a nonlinear elliptic equation in $\mathbb{R}^{2}$. Commun. Anal. Geom. 6, No.1, 1-19 (1998).

[13] B. Gidas, W-Y. Ni, L. Nirenberg. Symmetry and related properties via the maximum principle. Comm. Math. Phys. 68 (1979), no. 3, 209-243.

[14] J.M. Lee, T.H. Parker. The Yamabe problem. Bull.Amer.Math.Soc (N.S) 17 (1987), no.1, 37 -91.

[15] YY. Li. Prescribing scalar curvature on $\mathbb{S}_{n}$ and related Problems. C.R. Acad. Sci. Paris 317 (1993) $159-164$. Part I: J. Differ. Equations 120 (1995) 319-410. Part II: Existence and compactness. Comm. Pure Appl.Math.49 (1996) 541-597.

[16] YY. Li. Harnack Type Inequality: the Method of Moving Planes. Commun. Math. Phys. 200,421-444 (1999).

[17] YY. Li, L. Zhang. A Harnack type inequality for the Yamabe equation in low dimensions. Calc. Var. Partial Differential Equations 20 (2004), no. 2, 133-151.

[18] YY.Li, M. Zhu. Yamabe Type Equations On Three Dimensional Riemannian Manifolds. Commun.Contem.Mathematics, vol 1. No.1 (1999) 1-50.

[19] I. Shafrir. A sup+inf inequality for the equation $-\Delta u=V e^{u}$. C. R. Acad.Sci. Paris Sér. I Math. 315 (1992), no. 2, 159-164.

[20] Y-T. Siu. The existence of Kahler-Einstein metrics on manifolds with positive anticanonical line bundle and a suitable finite symmetry group. Ann. of Math. (2) 127 (1988), no. 3, 585-627

[21] G. Tian. A Harnack type inequality for certain complex Monge-Ampere equations. J. Differential Geom. 29 (1989), no. 3, 481-488. 
Departement de Mathematiques, Universite Pierre et Marie Curie, 2 place Jussieu, 75005, PARIS, FRANCE.

E-mail address: samybahoura@yahoo.fr 